\title{
Screening for anti-inflammatory components from Corydalis bungeana Turcz. based on macrophage binding combined with HPLC
}

\author{
Zi-Bo Dong ${ }^{1,2^{*}}$, Yong-Hong Zhang ${ }^{2}$, Bing-Jie Zhao ${ }^{2}$, Chao Li ${ }^{3}$, Gang Tian ${ }^{3}$, Ben Niu ${ }^{3}$, Hong Qi ${ }^{3}$, Liang Feng ${ }^{{ }^{*}}$ \\ and Jian-Guo Shao ${ }^{3}$
}

\begin{abstract}
Background: Corydalis bungeana Turcz. (CB; family: Corydalis DC.) is an anti-inflammatory medicinal herb used widely in traditional Chinese medicine (TCM) for upper respiratory tract infection, etc., but its anti-inflammatory active molecules are unknown. This study was designed to screen for the anti-inflammatory components from CB based on macrophage binding combined with HPLC.

Methods: Xylene-induced ear edema in mouse and carrageenan-induced hind-paw edema in rats were used to evaluate the anti-inflammatory activity of CB. The macrophage binding with high-performance liquid chromatography (HPLC) analysis and HPLC-MS were established to screen the potential active compounds. ELISA kits were performed to measure the levels of IL-6, IL-10, TNF- $a$ and NO in RAW 264.7 macrophages culture media.

Results: The alkaloid extract of CB could inhibit significantly xylene-induced ear edema in mouse and carrageenan-induced hind-paw edema in rats. Two components binded to RAW 264.7 cell were identified as 12-hydroxycorynoline and corynoline. Bioassays demonstrated that these two compounds significantly inhibited LPS-induced IL-6, IL-10, TNF-a and NO levels.

Conclusions: The results suggest that corynoline and 12-hydroxycorynoline contribute to the anti-inflammatory effects of the alkaloid extract of CB. Our findings suggest that these two compounds can be used as candidate for anti-inflammatory drugs.
\end{abstract}

Keywords: Corydalis bungeana Turcz., Anti-inflammatory, Macrophage, HPLC

\section{Background}

Corydalis bungeana Turcz. (CB; family: Corydalis) is a perennial herb containing several pharmacologically important alkaloids such as corydaline, 12-hydroxycorynoline, protopine, acetylcorynoline, and corynoline [1]. The dried whole plant is referred in traditional Chinese medicine (TCM) as Herba Corydalis Bungeanae, clearing heat and toxins, as well as anti-inflammatory [2]. Corynoline is the major alkaloid component derived from CB. It has been known that corynoline, acetylcorynoline, and protopine can significantly reduce carbon tetrachloride $\left(\mathrm{CCl}_{4}\right)$-induced microsomal lipid peroxidation [3] and attenuate two, 4-

\footnotetext{
*Correspondence: 13961098521@163.com; wenmoxiushi@163.com ${ }^{1}$ Post-doctoral Research Center, Nanjing University of Chinese Medicine \& Jumpcan Pharmaceuticl Co.,Ltd, Jiangsu, Taizhou 225441, P. R. China Full list of author information is available at the end of the article
}

dinitro-1-fluorobenzene-induced delayed-type hypersensitivity [2]. Some isoquinoline alkaloids such as 12hydroxycorynoline isolated from $\mathrm{CB}$ have bacteriostatic activity [4]. CB has been used for treating influenza, infections of the upper respiratory tract, bronchitis, tonsillitis, acute nephritis, and pyelonephritis [5]. However, the molecules responsible for treating inflammatory diseases have not been identified.

Extraction and purification of compounds from herbs is time-consuming. Screening of bioactive compounds for use in animal models of disease is also laborious and inappropriate for TCMs [6]. The action of drugs is usually demonstrated a fact that the drug molecules interacted with receptors, enzymes or channels on cell membranes, and then mediated signaling cascade [7]. A screening method involving in the binding of molecules 
to the membranes of human red blood cells and highperformance liquid chromatography (HPLC) analysis was developed to identify the active components of herb extract [8]. Here, we report an improved method based on macrophage binding and HPLC. Cell suspensions were treated with the alkaloid extract of $\mathrm{CB}$ and then centrifuged to remove unbound components. The final eluate was collected as a sample for screening potential active components in medicinal herbs by HPLC analysis.

Lipopolysaccharide (LPS) is an endotoxin and a constituent of the outer membrane of Gram-negative bacteria [9]. LPS-stimulated macrophages can produce various inflammatory mediators: interleukins (such as IL-6, IL-8, etc.), interferon (IFN)- $\gamma$ and TNF- $\alpha$ [10]. Levels of inflammatory mediators in the supernatants of LPS-stimulated macrophages are reliable markers of inflammation. Various chemicals in plants show anti-inflammatory activity by modulating levels of inflammation-associated genes and inhibiting the release of inflammation-associated mediators. Hence, stimulation of macrophages with LPS offers an excellent model for screening and subsequent evaluation of the inflammatory effects of candidate drugs.

In this study, we screened the components from the extract of $\mathrm{CB}$ and then evaluated the effects of components screened from the alkaloid extract of CB using LPSstimulated RAW 264.7 cells (a macrophage cell line) by examining cytokine production.

\section{Methods}

\section{Plant materials and reagents}

The samples of CB were purchased from Anhui Pharmaceutical Company (An'hui, China; voucher specimen ID number: 20120425). Botanic identification was confirmed by Professor Chungen Wang (Nanjing University of Chinese Medicine, Nanjing, China). Specimens are stored in the Herbal Specimens Center of Jumpcan Pharmaceuticl Co.,Ltd (Stored specimen No. JC-2012005). Protopine, 12-hydroxycorynoline, 6-acetonylcorynoline, corynoline and acetylcorynoline were prepared by the Nanjing University of Chinese Medicine and purity was $99 \%$ according to HPLC analysis. Aspirin was obtained from Nanjing Baijingyu Pharmaceutical Co., Ltd. (Nanjing, China). Xylene was purchased from Shanghai Qiangshun Pharmaceutical Co., Ltd. (Shanghai, China). LPS and carrageenan were obtained from Sigma-Aldrich (Saint Louis, MO, USA). Dulbecco's modified Eagle's medium (DMEM) was purchased from Gibco (Grand Island, NY, USA). Fetal bovine serum (FBS) was obtained from Wisent Biological Corporation (Nanjing, China). Enzyme-linked immunosorbent assay (ELISA) kits for determination of the levels of IL-6, IL-10 and TNF- $\alpha$ were purchased from eBioscience (San Diego, CA, USA). Kits for the measurement of nitric oxide $(\mathrm{NO})$ was obtained from Beyotime Institute of Biotechnology (Shanghai, China). The murine macrophage cell line (RAW 264.7 cells) was kindly provided by Professor Xu Qiang (Nanjing University, Nanjing, China). Water for HPLC analysis was purified using a Milli-Q Water Purification system (Millipore, Bedford, MA, USA). Other reagents were of analytical grade.

\section{Animals}

ICR mice (18-24 g) and Sprague-Dawley (SD) rats (150-200 g) were purchased from the Zhejiang Experimental Animal Center (Zhejiang, China). All animals were maintained at the Nanjing University of Chinese Medicine under specific pathogen-free conditions. The study protocol was authorized by the Ethics Committee of Nanjing University of Chinese Medicine (Nanging, China) in accordance with the Guide for the Care and Use of Laboratory Animals (National Institutes of Health, Bethesda, MD, USA).

\section{Extraction and isolation of alkaloids}

Air-dried herbs $(20 \mathrm{~kg})$ were extracted twice with 200 and $160 \mathrm{l}$ of $60 \%(\mathrm{v} / \mathrm{v})$ ethanol (blend $95 \%(\mathrm{v} / \mathrm{v})$ ethanol with appropriate water to $60 \%$ ) for $1 \mathrm{~h}$ successively. Extracts were collected and concentrated under reduced pressure at $55{ }^{\circ} \mathrm{C}$. Concentrates were suspended in water, adding $1 \mathrm{~mol} / \mathrm{L} \mathrm{HCl}$ to adjust $\mathrm{pH} 2$ of the solution, left for $4 \mathrm{~h}$, then $40 \%(\mathrm{v} / \mathrm{v}) \mathrm{NaOH}(40 \mathrm{~g} \mathrm{NaOH}$ dissolved in $100 \mathrm{ml}$ of water) mixed with the liquor until its $\mathrm{pH} 9$, left overnight. Extracts $(300 \mathrm{~g})$ were filtered to obtain a fraction of crude alkaloids and then extracted with a ten-fold volume of $95 \%(\mathrm{v} / \mathrm{v})$ ethanol under reflux for $1 \mathrm{~h}$. This extraction was repeated twice. The extract was collected and concentrated under reduced pressure at $55{ }^{\circ} \mathrm{C}$ (i.e., $20 \mathrm{~g}$ of alkaloid components with minimum polarity). The residue was extracted with equal volumes of butanol thrice and combined with butanol. The extract was collected and concentrated under reduced pressure at $55{ }^{\circ} \mathrm{C}$ (i.e., alkaloid components with maximum polarity). The samples (i.e., alkaloid extract of $\mathrm{CB}$ ) were ready for HPLC analysis.

\section{Cell culture and binding}

RAW 264.7 macrophages were cultured in DMEM supplemented with $10 \% \mathrm{FBS}$, antibiotics $(10 \mathrm{U} / \mathrm{mL}$ penicillin G, $100 \mathrm{~g} / \mathrm{mL}$ streptomycin), and maintained at $37{ }^{\circ} \mathrm{C}$ in a humidified incubator containing $5 \% \mathrm{CO}_{2}$. Cell suspensions $\left(10 \mathrm{~mL} ; 2 \times 10^{7}\right.$ cells) were treated with the alkaloid extract of $\mathrm{CB}(0.5 \mu \mathrm{g} / \mathrm{mL})$ for $1 \mathrm{~h}$ at $37^{\circ} \mathrm{C}$. Suspensions were then centrifuged for $15 \mathrm{~min}$ at $1000 \mathrm{rpm}(175 \times \mathrm{g})$ at room temperature and cell pellets were washed for ten times to remove unbound components. Washed eluates were discarded except for the final eluate, which was collected as a control for HPLC analysis (sample A). RAW 
264.7 macrophages were denatured by addition of $10 \mathrm{~mL}$ hydrochloric acid and phosphate-buffered saline (PBS; $\mathrm{pH} 4.0)$ to liberate components bound to cells whithout damaging the physiological state of cells. The desorption eluate was clarified by centrifugation and retained for HPLC analysis (sample B). Cells treated with dimethyl sulfoxide (DMSO) alone were used as the vehicle control of the alkaloid extract of $\mathrm{CB}$, as the vehicle desorption eluate (sample $\mathrm{C})$. Aliquots $(1 \mathrm{~mL})$ of samples $\mathrm{A}, \mathrm{B}$ and $\mathrm{C}$ were mixed separately with $1 \mathrm{~mL}$ methanol, vortex-mixed for $2 \mathrm{~min}$, and centrifuged at 10,000 rpm for $3 \mathrm{~min}$ at room temperature. Supernatants were collected and dried under nitrogen at $45{ }^{\circ} \mathrm{C}$. Residues were taken up into $200 \mu \mathrm{L}$ methanol and filtered through a $0.45-\mu \mathrm{m}$ nylon membrane before HPLC analysis.

\section{HPLC analysis}

The alkaloid extract of CB and RAW 264.7 macrophagebound samples were analyzed by HPLC. We used a Series 1100 Liquid Chromatograph (Agilent Technologies, Palo Alto, CA, USA) equipped with a vacuum degasser, a quaternary pump, an autosampler and a photodiode-array detector (PDA) connected to Agilent ChemStation software. A phenomenex Gemini C18 ODS column (4.6 $\mathrm{mm} \times$ $250 \mathrm{~mm}, 5 \mu \mathrm{m}$ ) was used. The mobile phase was (A) ammonium acetate (10 mM; pH 9.5); (B) methanol. The flow rate was $1 \mathrm{~mL} / \mathrm{min}$. The elution conditions were: $\mathrm{B}, 0-$ 45 min, linear gradient $15-60 \%$ B; 45-70 min, linear gradient $60-86 \%$ B; 70-80 min, linear gradient 86-15\% B. The system operated at $30{ }^{\circ} \mathrm{C}$ and the injection volume was $10 \mu \mathrm{L}$. The detection wavelength was kept at $289 \mathrm{~nm}$.

\section{HPLC-mass spectrometry (MS) analysis}

MS conditions were: positive ion model; drying gas, $\mathrm{N}_{2}$; spray tip potential, $5290 \mathrm{~V}$; nozzle potential, $140 \mathrm{~V}$; nozzle temperature, $140{ }^{\circ} \mathrm{C}$; detector voltage, $2100 \mathrm{~V}$; scan range, 50-1000 m/z. An Alliance $2690 \mathrm{HPLC}$ instrument (Waters, Milford MA, USA) coupled with a UV detector was used for quantitative determination. The chromatographic conditions were as described above. Chromatograms were monitored at $289 \mathrm{~nm}$. The structure was confirmed by $\mathrm{H}-\mathrm{NMR}$ and $\mathrm{C}^{13}$-NMR. (5 mg sample was dissolved in $\mathrm{d} 6-\mathrm{CDCl}_{3}$, and detected by AV-500. BRUKER.)

\section{Evaluation of anti-inflammatory activity in vitro}

RAW 264.7 macrophages $\left(1.2 \times 10^{6}\right)$ were cultured in DMEM supplemented with $10 \%$ FBS and maintained at $37{ }^{\circ} \mathrm{C}$ in a humidified incubator containing $5 \%$ $\mathrm{CO}_{2}$. Cells were stimulated with LPS $(0.5 \mu \mathrm{g} / \mathrm{mL})$ for $24 \mathrm{~h}$ and treated with different concentrations $(100 \mu \mathrm{g} / \mathrm{mL}, 10 \mu \mathrm{g} / \mathrm{mL}, 1 \mu \mathrm{g} / \mathrm{mL}, 100 \mathrm{ng} / \mathrm{mL}$ and $10 \mathrm{ng} / \mathrm{mL}$ ) of compounds obtained from the alkaloid extract of CB for $1 \mathrm{~h}$. Control values were obtained in the absence of LPS and compounds. Levels of IL-6, IL$1 \beta$, NO and TNF- $\alpha$ in macrophage culture media were measured by ELISA kits according to manufacturer's instructions.

\section{Xylene-induced ear edema in mice}

The xylene-induced ear edema test with modifications was conducted as described previously [11]. Briefly, four groups of ten mice were treated (via the oral route) with physiological (0.9\%) saline (control), alkaloid extract of CB (40 and $80 \mathrm{mg} / \mathrm{kg}$ ) or aspirin (150 mg/kg; positive control). Mice were co-administrated (by gavage) with different doses of the alkaloid extract of $\mathrm{CB}$ intragastrically once a day for 5 days. About $1 \mathrm{~h}$ after administration, each mouse received $50 \mu \mathrm{L}$ of xylene on the inner and outer surfaces of the right ear. A further 1-h later, ear thickness was measured with a Thickness Gauge (Harbin Measuring \& Cutting
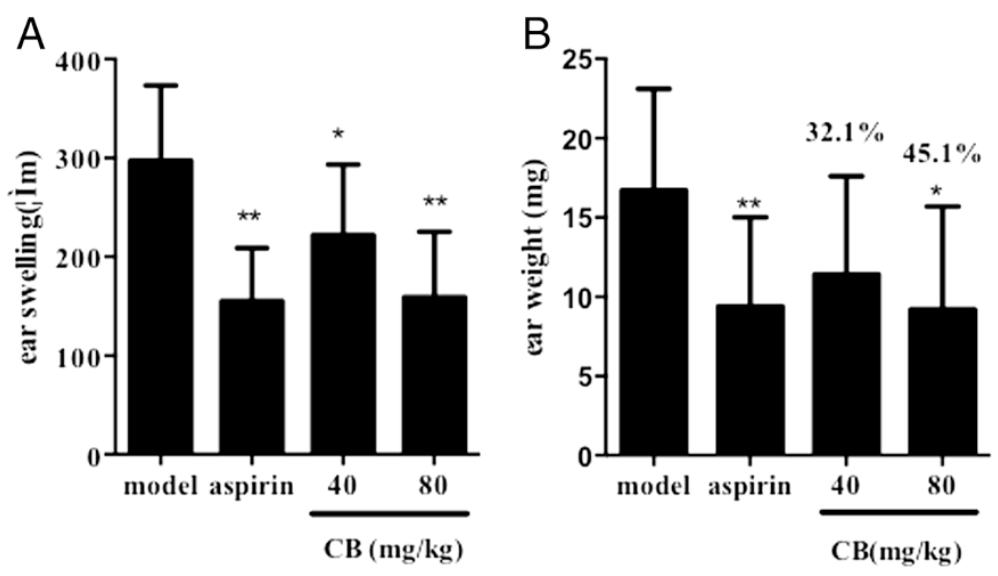

Fig. 1 Anti-inflammatory effect of the alkaloid extract of CB on ear swelling (a) and ear weight (b) in xylene-induced mice. Values are expressed as mean $\pm \mathrm{SD}(n=10) .{ }^{*} p<0.05$ and ${ }^{* *} p<0.01$ vs. model group 


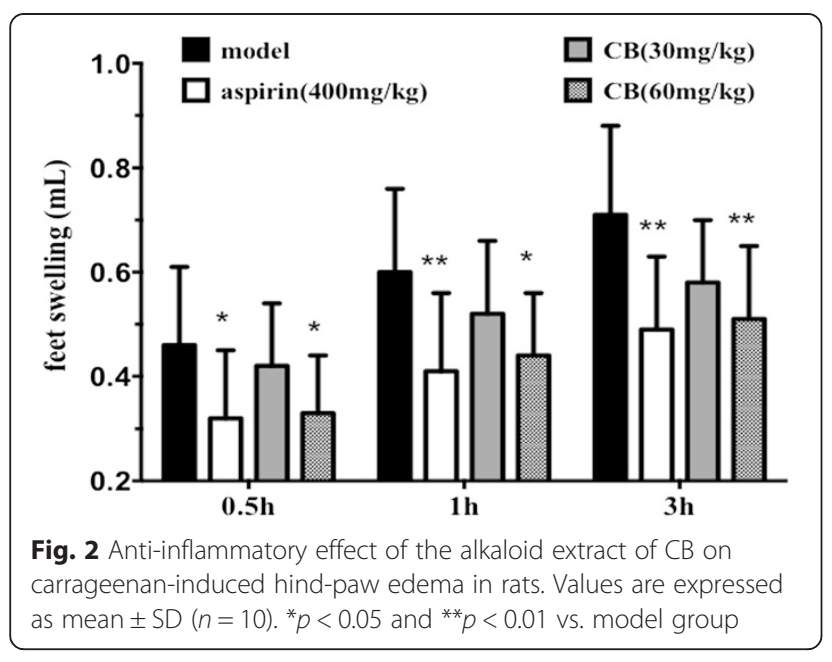

Tool Group Co., Ltd., Harbin, China). Mice were killed by cervical dislocation. Ear biopsies (diameter, $9.0 \mathrm{~mm}$ ) were punched out and weighed. The degree of ear swelling was calculated based on the weight difference between the biopsies of the right and left ear of the same mouse. Percent inhibition of edema was calculated using the formula:

$$
\begin{aligned}
\text { Edema inhibition }(\%)= & \left(\text { edema }_{\text {control }^{-}} \text {edema }_{\text {drugs }}\right) \\
& / \text { edema }_{\text {control }} \times 100 \%
\end{aligned}
$$

\section{Carrageenan-induced paw edema in rats}

The method used for this investigation was similar to that reported previously [11]. Forty male SD rats were divided into four groups: model control (saline); alkaloid extract of CB $(30 \mathrm{mg} / \mathrm{kg}$ and $60 \mathrm{mg} / \mathrm{kg})$; aspirin $(400 \mathrm{mg} / \mathrm{kg})$.

The normal volume of the right hind-paw of each rat was measured with a Water Displacement Plethysmometer (YLS-7B; YiyanTechnology Co, Ltd, Jinan, China) after the final administration of extract. Then, each rat was treated with $0.1 \mathrm{~mL}$ of $1 \%$ carrageenan by subcutaneous injection into the right hind-paw $1 \mathrm{~h}$ after administration. Paw volume was measured again at 0.5 , 1 and $3 \mathrm{~h}$ after the induction of inflammation. Swelling volume was calculated as $V_{2}-V_{1}$, where $V_{1}$ and $V_{2}$ are the right hind-paw volumes $(\mathrm{mL})$ of the first and second measurements, respectively.

\section{Statistical analysis}

Data are expressed as the mean $\pm \mathrm{SD}$. Comparisons between groups were analyzed by one-way ANOVA. Dunnett's test was employed for comparison between two groups. $p<0.05$ was considered significant.

\section{Results}

Effect of the alkaloid extract of $C B$ on xylene-induced ear edema in mice

Utilizing a typical model of inflammatory response (xylene-induced ear edema), the anti-inflammatory activity of the alkaloid extract of $\mathrm{CB}$ was investigated.

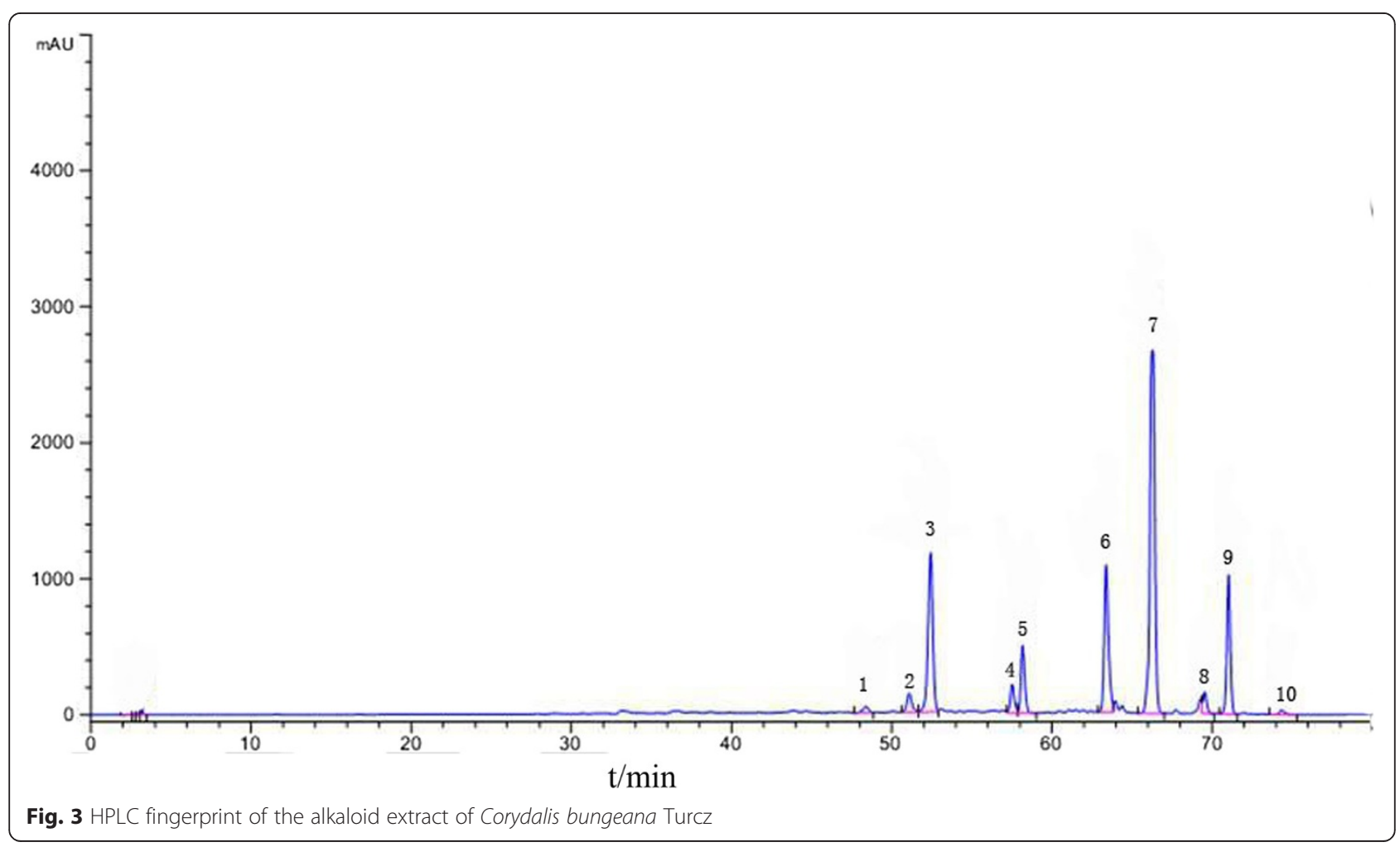


Table 1 RRT and the RPA of each peak in the fingerprint of the alkaloid extract of CB

\begin{tabular}{llrl}
\hline Peak number & RRT & \multicolumn{1}{l}{ RPA } & Identified compound \\
\hline 1 & 1.370 & 47.444 & unknown \\
2 & 1.297 & 19.862 & unknown \\
3 & 1.264 & 2.367 & Protopine \\
4 & 1.152 & 15.868 & unknown \\
5 & 1.139 & 6.923 & 12-hydmxycorynoline \\
6 & 1.046 & 2.849 & 6-acetonylcorynoline \\
7 & 1.000 & 1.000 & Corynoline \\
8 & 0.953 & 21.822 & Acetylcorynoline \\
9 & 0.933 & 3.622 & unknown \\
10 & 0.892 & 75.680 & unknown \\
\hline
\end{tabular}

The alkaloid extract of CB (40 and $80 \mathrm{mg} / \mathrm{kg}$ ) showed significant inhibition of xylene-induced mice ear edema as compared with the control, with inhibition of 32.1 and $45.1 \%(P<0.01)$ respectively (Fig. 1$)$.

\section{Effect of the alkaloid extract of CB on carrageenan- induced hind-paw edema}

Carrageenan-induced paw inflammation is an acute and highly reproducible model of acute inflammatory response [12]. Cardinal signs of inflammation develop immediately after subcutaneous injection and result from action of pro-inflammatory agents. The inflammatory response is usually quantified by increase in paw size (edema) and is modulated by inhibitors within the inflammatory cascade [13]. A bar chart is given to illustrate the anti-inflammation effect of the alkaloid extract of $\mathrm{CB}$ in Fig. 2. The results indicate that the swelling volume increased progressively and peaked at 3rd hour after carrageenin injection. However, the edema of rat paw was significantly reduced by the treatment with the high-dose alkaloid extract of CB $(60 \mathrm{mg} / \mathrm{kg})$ during all phases of carrageenan-induced inflammation. And the inhibition ratios of the $\mathrm{CB}(60 \mathrm{mg} / \mathrm{kg})$ treatment with rats at $0.5,1$ and $3 \mathrm{~h}$ were $29.8 \%(P<0.05), 28.3 \%(P<$ $0.05)$ and $28.6 \%(P<0.01)$, respectively, compared to the model control. Aspirin was used as a positive control and had similar effects (31.9, 32.5 and $30.0 \%$ inhibition, respectively).

\section{HPLC analysis for the alkaloid extract of CB}

First, we established the fingerprint of the alkaloid extract of $\mathrm{CB}$ as the background information of macrophage binding. There were ten main peaks in the fingerprint of the alkaloid extract of $C B$ at $289 \mathrm{~nm}$ (Fig. 3). Among these, five components were determined after comparison with standards (Table 1). All of the main peaks had good separation.

Injection precision was determined by five replicate injections of the same sample in one day. The relative standard deviations (RSDs) of relative retention time (RRT) and relative peak area (RPA) were 0.72 and $0.56 \%$, respectively, and the changes were not significant. Repeatability was assessed by analyzing five independently prepared samples of the alkaloid extract of CB. RSDs of RRT and RPA were $1.22,1.57 \%$, respectively, and the changes were not significant. Sample stability was assessed by successive injections of the same sample at $0,1,2,4,8,12$ and $24 \mathrm{~h}$. During this period, the solution was stored at room temperature. RSDs of RRT and RPA were 1.72 and $2.05 \%$, respectively, and the changes were not significant. Results of injection precision, repeatability and the stability test suggested that this method was adequate, valid and applicable. RRT and RPA of each characteristic principle was calculated using corynoline as the reference peak. The RRT and RPA of the ten peaks are shown in Table 1.

\section{Binding of the alkaloid extract of CB to RAW 264.7 cells}

The alkaloid extract of $\mathrm{CB}$ exerted anti-inflammatory properties, so we used its binding to RAW 264.7 cells to screen the active compounds within it. The alkaloid extract of CB was incubated with RAW 264.7 cells and bound components collected by centrifugation. After thoroughly washing and eluting from cell pellets, retained components were injected into HPLC system for analysis.

Two principal peaks (K-1 and K-2) were detected in sample B (desorption eluate of the alkaloid extract of CB) (Fig. 4a). No comparable peaks were detected in the chromatograms of the two control samples (sample A,

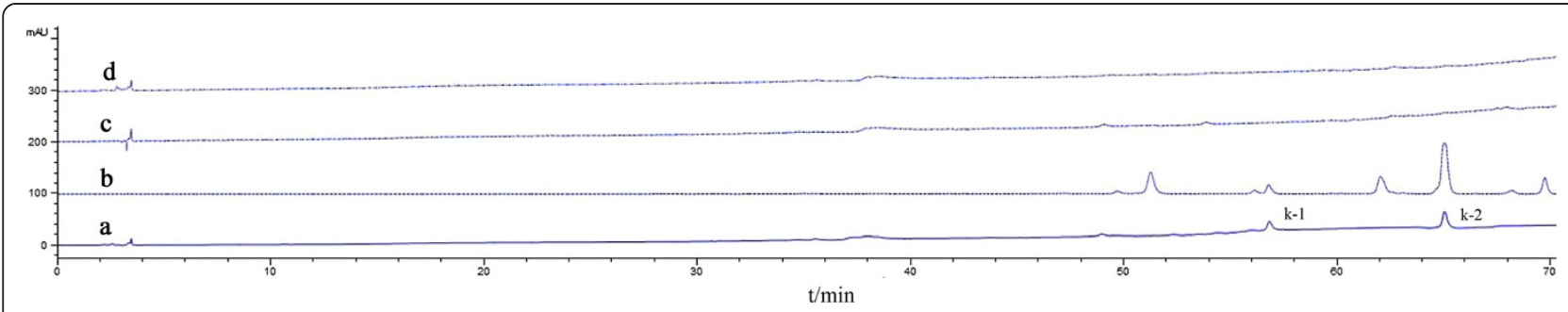

Fig. 4 Detection of RAW 264.7 cells-binding molecules in the alkaloid extract of CB by HPLC analysis. a Comparative chromatograms of the desorption eluate of alkaloid extract of $\mathrm{CB}$. $\mathbf{b}$ the fingerprint of the alkaloid extract of $\mathrm{CB}$. $\mathbf{c}$ the final wash eluate and $\mathbf{d}$ the vehicle desorption eluate. Detection was at $289 \mathrm{~nm}$ 
final wash eluate; sample $\mathrm{C}$, vehicle desorption eluate) (Fig. 4c and d). By comparison of the retention times and UV absorption profiles of $\mathrm{K}-1$ and $\mathrm{K}-2$ with the HPLC fingerprint of the alkaloid extract of $\mathrm{CB}$, components $\mathrm{K}-1$ and $\mathrm{K}-2$ were identified as peaks 2 and 3 (Fig. 4b). These findings identified the RAW 264.7 cell- binding molecules $\mathrm{K}-1$ and $\mathrm{K}-2$ as potential antiinflammatory components of $\mathrm{CB}$.

Identification of K-1 and K-2 by HPLC-MS

$5 \mathrm{mg}$ of $\mathrm{K} 1$ and $\mathrm{K} 2$ were respectively dissolved in $\mathrm{d} 6$ $\mathrm{CDCl}_{3}$ as the samples, and detected by Bruker AV-500.

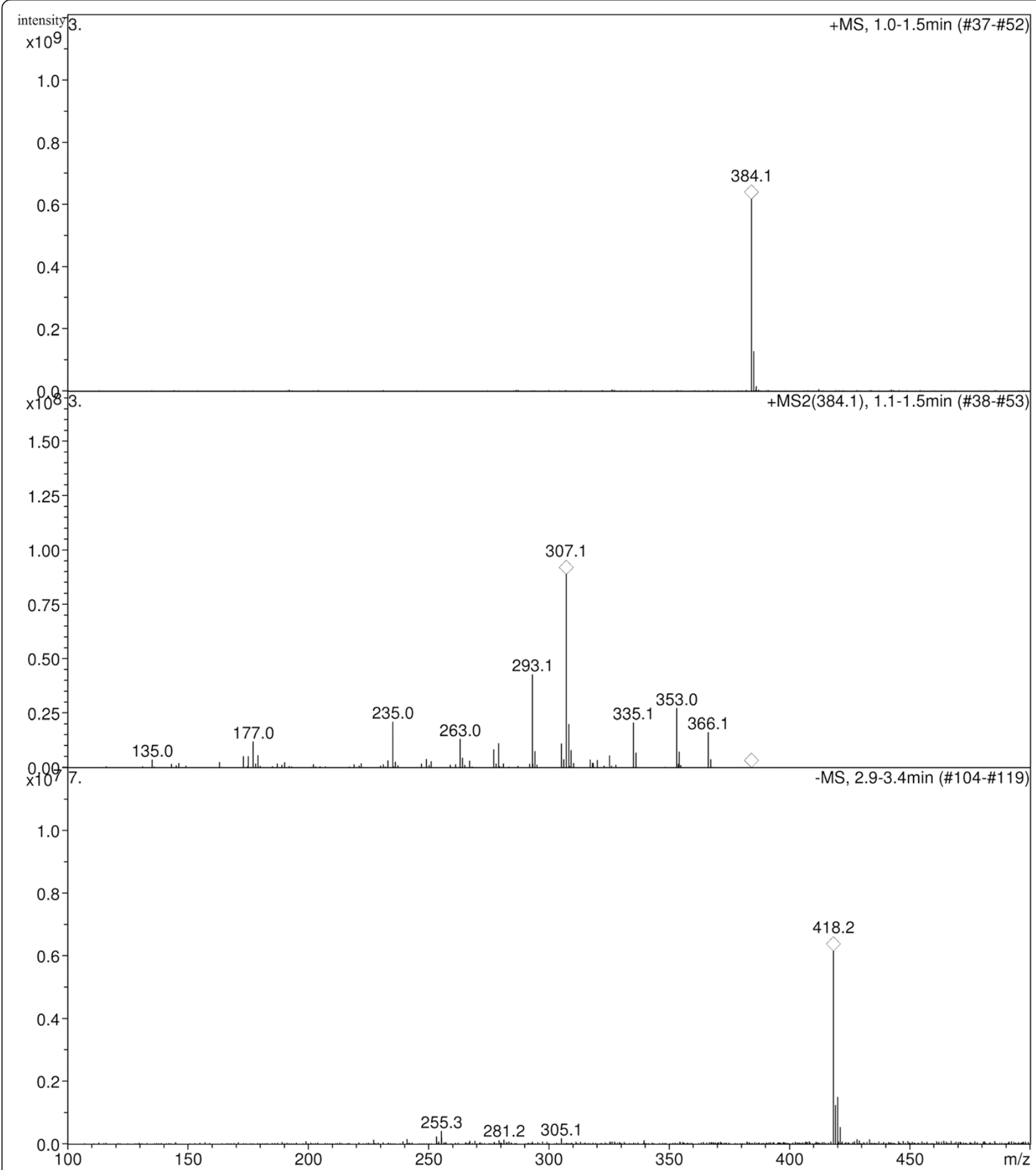

Fig. 5 ESI-MS negative ionization spectrum of K-2 obtained from macrophage binding 
The MS spectrum of compound K-2 is shown in Fig. 5. The mass spectrum of compound $\mathrm{K}-2$ was similar to the mass spectrum of 12-hydoxycorynoline. Identification was supported further by ${ }^{1} \mathrm{H}-\mathrm{NMR}$ data and ${ }^{13} \mathrm{C}$ NMR data: ${ }^{1} \mathrm{HNMR}(500 \mathrm{MHz}, \mathrm{CDCl} 3): 6.661(1 \mathrm{H}, \mathrm{s}$, 1-H), 7.023 (1H, s, 4-H), 2.177 (3H, s, 5-N-CH3), 3.437, $4.020(2 \mathrm{H}, \mathrm{ABq}, \mathrm{J}=15.5,6-\mathrm{H}), 6.808(1 \mathrm{H}, \mathrm{d}, \mathrm{J}=8.0$, 9$\mathrm{H}), 6.962(1 \mathrm{H}, \mathrm{d}, \mathrm{J}=8.0,10-\mathrm{H}), 3.878(1 \mathrm{H}, \mathrm{s}, 11-\mathrm{H})$, $4.902(1 \mathrm{H}, \mathrm{s}, 12-\mathrm{H}), 1.242(3 \mathrm{H}, \mathrm{s}, 13-\mathrm{CH} 3), 3.310(1 \mathrm{H}$, s, $14-\mathrm{H}), 5.995 \sim 5.942(4 \mathrm{H}, \mathrm{m}, 2-\mathrm{O}-\mathrm{CH} 2-\mathrm{O}-3,7-\mathrm{O}-$ CH2-O-8); ${ }^{13} \mathrm{C}-\mathrm{NMR}$ (125 MHz, CDCl3): 107.8 (C-1), 125.9 (C-1a), 145.1 (C-2), 147.0 (C-3), 112.0 (C-4), 128.8 (C-4a), 43.1 (5-N-CH3), 54.0 (C-6), 116.3 (C-6a), 142.7 (C-7), 148.6 (C-8), 109.9 (C-9), 118.9 (C-10), 135.5 (C-10a), 74.0 (C-11), 81.4 (C-12), 39.7 (C-13), 23.7 (13-CH3), 70.2 (C-14), 101.4 (2-O-CH2-O-3), 101.4 (9-O-CH2-O-10).

Compound $\mathrm{K}-1$ was postulated to be corynoline. Under identical chromatographic conditions, the minor polar compounds of $\mathrm{K}-1$ and the retention time of the reference peak of corynoline were identical.

\section{Anti-inflammatory activity of corynoline and 12-hydroxycorynoline in vitro}

ELISA assay was applied to examine the effect of corynoline and 12-hydroxycorynoline on IL-6, TNF- $\alpha$, IL-10 and NO levels in LPS-treated cells. As showed in Figs. 6 and 7, LPS alone dramatically increased the levels of IL-6, TNF- $\alpha$, IL-10 and NO. However, corynoline or 12-hydroxycorynoline at the certain concentrations obviously inhibited LPS-induced IL- 6 and TNF- $\alpha$ overproduction $(P<0.01$ or $P<0.05)$. Corynoline $(100 \mu \mathrm{g} /$ $\mathrm{mL}$ ) significantly suppressed LPS-induced IL-10 overproduction $(P<0.05)$ while 12 -hydroxycorynoline had no obvious effect on this index (Fig. 7a). In addtion, Corynoline $(1,100 \mu \mathrm{g} / \mathrm{mL})$ and 12-hydroxycorynoline $(100 \mu \mathrm{g} / \mathrm{mL})$ significantly inhibited LPS-induced NO production (Fig. 7b).
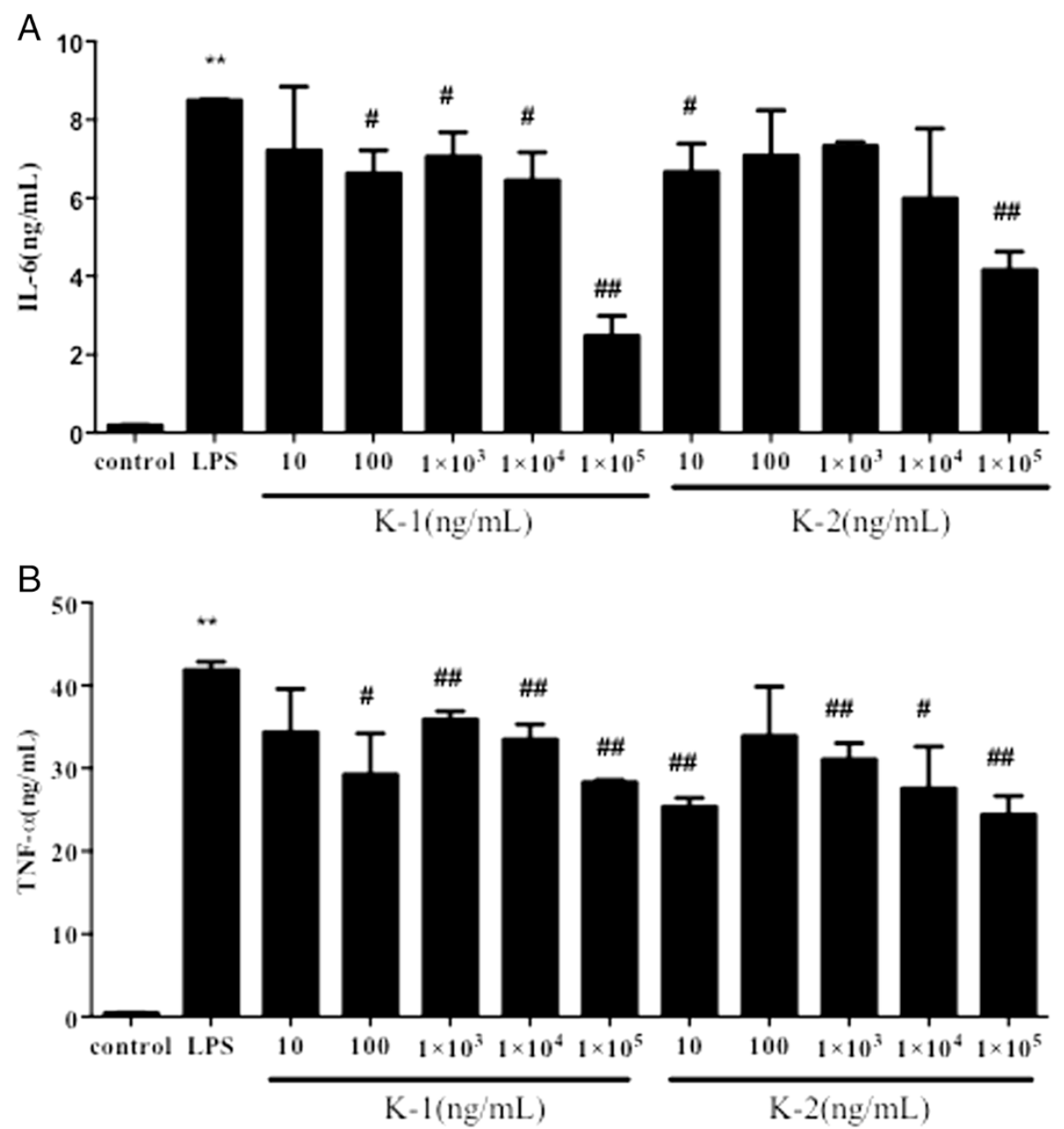

Fig. 6 Anti-inflammatory effect of corynoline and 12-hydroxycorynoline on the level of IL-6 (a) a nd TNF-a (b) induced by LPS in RAW 264.7 cells. Data are expressed as mean \pm SD $(n=10) .{ }^{*} p<0.05$ and ${ }^{* *} p<0.01$ vs. model group 

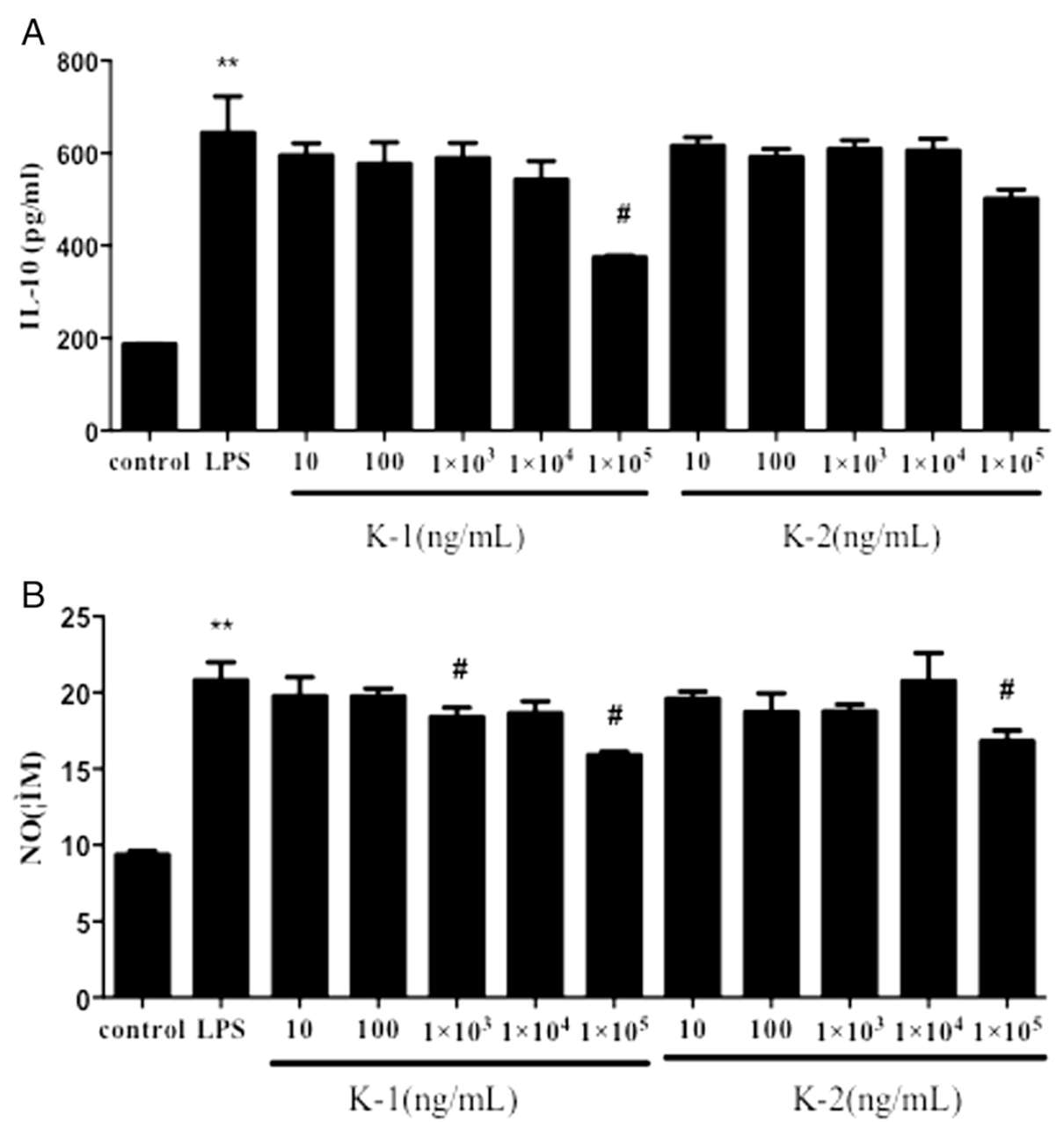

Fig. 7 Anti-inflammatory effect of corynoline and 12-hydroxycorynoline on the level of IL-10 (a) and NO (b) induced by LPS in RAW 264.7 cells. Data are expressed as mean \pm SD $(n=10) .{ }^{*} p<0.05$ and ${ }^{* *} p<0.01$ vs. model group

\section{Discussion}

$\mathrm{CB}$, a perennial herb, has been shown to possess multiple biological activities, such as anti-bacterial and anti-viral. Its extract contains abundant alkaloids which are responsible for the potential pharmacological activity and been used as heat-clearing and detoxifying agent. However, there is little knowledge about the anti-inflammatory activity and the potential anti-inflammation components. In the present study, we used aspirin as positive antiinflammatory compound because it is recognized as antipyretic analgesics. Compared with it, we found that the total alkaloids of CB have a significant anti-inflammatory activity in vitro and in vivo. More importantly, compounds $\mathrm{K}-1$ and $\mathrm{K}-2$ bound to macrophages which have been identified as 12-hydmxycorynoline and corynoline can inhibit LPS-induced inflammatory responses in RAW 264.7 cells.

The interesting and significant efficacy of herbs or TCM has aroused the increasing interest of many researchers. However, existing research is very difficult due to its complex components. The active ingredient in Chinese medicine and herbal medicine has been the focus of research. Cells membrane chromatography (CMC), a chromatographic biological affinity method that uses specific cell membranes as the stationary phase, has been confirmed as a simple, specific, effective screening method based on the binding principle that the recognition and interaction of drug molecules and targets, receptors, channels on the cell membrane [14]. A novel peritoneal macrophage/cell membrane chromatography (PM/CMC)-HPLC/MS method was shown to be an effective screening system for the rapid detection, enrichment, and identification of anti-inflammatory components from TCM [14]. Herein, we carried out this effective screening method to screen antiinflammatory components from CB. The structure identification by spectral and chromatographic techniques provided the evidence that corynoline and 12hydmxycorynoline are the binding components to RAW 264.7 cells. Their anti-inflammatory activities 
were proved in our study. This study supported the notion that the binding of RAW 264.7 cells combined with HPLC provides a rapid and efficient method for the identification of potential anti-inflammatory components in complex mixtures derived from medicinal herbs used in TCM.

Endotoxin LPS are the major molecular component of the outer membrane of Gram-negative bacteria and may excite the local inflammatory response [15]. The inhibition of LPS-induced pro-inflammatory cytokines from macrophages can be archived by LPS immobilization on porous and non-porous [16]. The stimulation of LPS could cause inflammatory response and regulate the release of inflammatory mediators. The potential inhibition of components on LPS-induced inflammatory response has been used to evaluate the anti-inflammatory activity.

Corynoline is the major alkaloid component derived from CB. Corynoline, acetylcorynoline, and protopine can significantly reduce $\mathrm{CCl}_{4}$-induced microsomal lipid peroxidation and attenuates 2,4-dinitro-1-fluorobenzene-induced delayed-type hypersensitivity. Some isoquinoline alkaloids such as 12-hydroxycorynoline isolated from CB hold potential bacteriostatic activity. LPS stimulates innate immunity by regulating the production of inflammatory mediators such as NO, TNF- $\alpha$, IL-6, prostanoids, and leukotrienes [17]. For example, IL-6 overexpression is involved in rheumatoid arthritis [18]. TNF- $\alpha$ exhibits its pro-inflammatory activity by regulating several intercellular and vascular cell adhesion molecules, which results in the recruitment of leukocytes to inflammation sites [19]. In vitro bioassays have demonstrated that corynoline and 12-hydroxycorynoline in CB significantly inhibit the production of certain inflammatory mediators, especially TNF- $\alpha$ and IL-6. It has been suggested that corynoline and 12-hydroxycorynoline contribute to the anti-inflammatory effects of CB.

Overall, the present study demonstrated the efficacy of the alkaloid extract of $\mathrm{CB}$ in different anti-inflammatory tests. Our established screening system showed that 12hydroxycorynoline and corynoline were two important active components for the inhibition of LPS-stimulated inflammatory responses. The anti-inflammatory effects of $\mathrm{CB}$ might be due at least in part to the corynoline and 12-hydroxycorynoline present in the plant. These two compounds might be regarded as potential candidate molecules for inflammation inhibitors.

\section{Conclusions}

Our experimental results indicated that corynoline and 12-hydroxycorynoline contribute to the antiinflammatory effects of the alkaloid extract of CB. Our findings also suggested that these two compounds can be used as candidate for anti-inflammatory drugs.

\section{Competing interests}

Jumpcan Pharmaceuticl Co., Ltd provided funding, design, implementation and analysis for this study. Nanjing University of Chinese Medicine performed efficacy assessments.

\section{Authors' contributions}

Z-BD and LF carried out the cell experiment studies and drafted the manuscript. $\mathrm{CL}$ and GT carried out the structure identification of the isolated compounds and analysis of extract. BN and HQ participated in animal activity evaluate. $\mathrm{Y}-\mathrm{HZ}$ and Z-BD conceived of the study and performed the statistical analysis. J-GS and Z-BD participated in the design of the study and coordination. B-JZ and Z-BD participated in the review of the manuscript. All authors read and approved the final manuscript. Jumpcan Pharmaceuticl Co. Ltd provided funding, design, implementation and analysis for this study. Nanjing University of Chinese Medicine performed efficacy assessments.

\section{Acknowledgments}

The authors were truly grateful to the financial support by the National Natural Science Foundation Project $(81202906,81473394)$ and High-level talent innovation of Jiangsu province (BRA2013144).

\section{Author details}

${ }^{1}$ Post-doctoral Research Center, Nanjing University of Chinese Medicine \& Jumpcan Pharmaceuticl Co.,Ltd, Jiangsu, Taizhou 225441, P. R. China. ${ }^{2}$ Nanjing University of Chinese Medicine, Jiangsu, Nanjing 210046, P. R. China. ${ }^{3}$ Jumpcan Pharmaceuticl Co., Ltd, Jiangsu, Taizhou 225441, P. R. China.

Received: 7 February 2015 Accepted: 8 October 2015

Published online: 15 October 2015

\section{References}

1. Niu L, Xie Z, Cai T, Wu P, Xue P, Chen X, et al. Preparative isolation of alkaloids from Corydalis bungeanaTurcz. by high-speed counter-current chromatography using stepwise elution. J Sep Sci. 2011;34(9):987-94.

2. Fu RH, Wang YC, Liu SP, Chu CL, Tsai RT, Ho YC, et al. Acetylcorynoline impairs the maturation of mouse bone marrow-derived dendritic cells via suppression of $1 \mathbf{k B}$ kinase and mitogen-activated protein kinase activities. PLoS One. 2013;8(3):e58398.

3. Wei HL, LiU GT. Protective action of corynoline, acetylcorynolineand protopine against experimental liver injury in mice. Yao Xue Xue Bao. 1997;32(5):331-6.

4. Zheng WG, Li WZ, Tang GS. Chemical Study of Alkaloids from Corydalis bungeana. Planta Med. 1987;53(5):418-20.

5. Chinese Pharmacopoeia Commission. Pharmacopoeia of the People's Republic of China (English Edition). Beijing: Chemical Industry Press; 2000. p. A-26

6. Jiang Y, David B, Tu P, Barbin Y. Recent analytical approaches in quality control of traditional Chinese medicines-a review. Anal Chim Acta. 2010;657(1):9-18.

7. Dong ZB, Li SP, Hong M, Zhu Q. Hypothesis of potential active components in Angelica sinensis by using biomembrane extraction and high performance liquid chromatography. J Pharm Biomed Anal. 2005;38(4):664-9.

8. Zheng ZG, Duan T, He B, Tang D, Jia XB, Wang RS, et al. Macrophage biospecific extraction and HPLC-ESI-MSn analysis for screening immunological active components in Smilacis Glabrae Rhizoma. J Pharm Biomed Anal. 2013;77:44-8.

9. Shen $L$, Li Y, Jiang $L$, Wang $X$. Response of Saccharomyces cerevisiae to the Stimulation of Lipopolysaccharide. PLoS One. 2014;9(8):e104428.

10. Hua KF, Yang TJ, Chiu HW, Ho CL. Essential oil from leaves of Liquidambar formosana ameliorates inflammatory response in lipopolysaccharide-activated mouse macrophages. Nat Prod Commun. 2014;9(6):869-72.

11. Niu X, Li Y, Li W, Hu H, Yao H, Li H, et al. The anti-inflammatory effects of Caragana tangutica ethyl acetate extract. J Ethnopharmacol. 2014;152(1):99-105.

12. Morris CJ. Carrageenan-induced paw edema in the rat and mouse. Methods Mol Biol. 2003;225:115-21.

13. Teng K, Ruan HS, Zhang HF. Antinociceptive and anti-inflammatory effects of roots extracts from Actinidiaarguta (Sieb. e t Zucc.) Planch. Pharmacognosy J. 2014;6(2):1-7.

14. Li W, Xing W, Wang S, Fan T, Huang H, Niu X, et al. An online coupled peritoneal macrophage/cell membrane chromatography and high-performance liquid chromatography/mass spectrometry method to screen for anti-inflammatory 
components from the Chinese traditional medicine Chloranthus multistachys Pei. Biomed Chromatogr. 2013;27(11):1580-6.

15. Tous M, Ferrer-Lorente R, Badimon L. Selective inhibiton of Sphingosine kinase-1 protects adipose tissue against LPS-induced inflammatory response in Zucker diabetic fatty rats. Am J Physiol Endocrinol Metab. 2014;307(5):E437-446.

16. López-Abarrategui C, Del Monte-Martínez A, Reyes-Acosta O, Franco OL, Otero-González AJ. LPS inmobilization on porous and non-porous supports as an approach for the isolation of anti-LPS host-defense peptides. Front Microbiol. 2013;4:389.

17. Opal SM. The host response to endotoxin, antilipopolysaccharidestrategied, and the management of severe sepsis. Int J Med Microbiol. 2007;297(5):365-77.

18. Tanaka T, Narazaki M, Ogata A, Kishimoto T. A new era for the treatment of inflammatory autoimmune diseases by interleukin-6 blockade strategy. Semin Immunol. 2014;26(1):88-96.

19. Leick M, Azcutia V, Newton G, Luscinskas FW. Leukocyte recruitment in inflammation: basic concepts and new mechanistic insights based on new models and microscopic imaging technologies. Cell Tissue Res. 2014;355(3):647-56.

\section{Submit your next manuscript to BioMed Central and take full advantage of:}

- Convenient online submission

- Thorough peer review

- No space constraints or color figure charges

- Immediate publication on acceptance

- Inclusion in PubMed, CAS, Scopus and Google Scholar

- Research which is freely available for redistribution 